\title{
Optical alignment, characterization, and testing through image-based output beam analysis: application to Sentinel 5 OGSE
}

R. Henriques, J. K. Abrantes, J. Mendes-Lopes

R. Henriques, J. K. Abrantes, J. Mendes-Lopes, "Optical alignment, characterization, and testing through image-based output beam analysis: application to Sentinel 5 OGSE," Proc. SPIE 11852, International Conference on Space Optics - ICSO 2020, 118524D (11 June 2021); doi:

$10.1117 / 12.2599652$

SPIE Event: International Conference on Space Optics - ICSO 2021, 2021, Online Only 


\section{International Conference on Space Optics-ICSO 2020}

Virtual Conference

30 March-2 April 2021

Edited by Bruno Cugny, Zoran Sodnik, and Nikos Karafolas
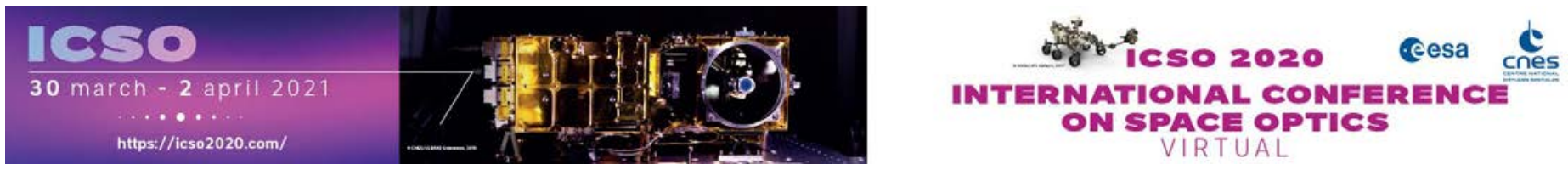

\section{Optical alignment, characterization, and testing through image- based output beam analysis: application to Sentinel 5 OGSE}

\section{Cesa isoporacedings ecnes}




\title{
Optical Alignment, Characterization and Testing through Image- Based Output Beam Analysis: Application to Sentinel 5 OGSE
}

\author{
R. Henriques*a, J. K. Abrantes ${ }^{\mathrm{a}}$, J. Mendes-Lopes ${ }^{\mathrm{a}}$ \\ ${ }^{a}$ Lusospace, Rua Sarmento de Beires 31A, Lisbon, Portugal, 1900-411
}

\begin{abstract}
An image-based method used for optical alignment, characterization and testing of the Sentinel 5 AIT OGSE output beams is described here. The OGSEs will be used in the AIT campaign of the Sentinel 5 spectrograph instruments, and each of them comprises two modules assembled in a common mechanical base, one generating point sources and the other an astigmatic optical beam.

The methods described in the present work evaluate optical beam parameters such as the spatial and spectral F-numbers, relative beam pointing (rotations about $X$ and $Y, Z$ being the optical axis) and astigmatic beam parameters, among others. Such evaluation is made possible through the acquisition and processing of series of images along the optical axis, by scanning a CCD module with controlled small displacements $\Delta Z$ imposed by a precision motorized linear stage.

Special attention is given in this paper to the alignment of the optical components of the astigmatic module of the OGSEs, performed with the aid of described method. To this end, the astigmatic beam parameters to be optimized - spatial and spectral F-numbers, relative pointing, and astigmatism - are described as a function with linear dependence on each of the system opto-mechanical degrees of freedom, considering small variations around their nominal values. These dependencies can be described by a set of equations arranged in matrix form. Solution of the linear system then provides a direct indication of the dimensional adjustments that must be imposed to each degree of freedom in order to align the optical system from a set of initial values. To calculate each coefficient of the matrix $M$, the sensitivity of the dependent variables with each of the actuators was previously simulated in raytracing software Zemax OpticStudio.
\end{abstract}

The overall methodology was successfully applied to the alignment phase of the set of OGSEs, with accuracies - based on actual experimental repeatability - of \pm 0.02 for spatial and spectral F-numbers, $\pm 10 \mu \mathrm{m}$ for the distance between foci of the astigmatic beam, and \pm 0.2 arcmin for relative beam pointing.

Keywords: AIT, OGSE, Astigmatic beam, Optical Metrology.

\section{INTRODUCTION}

The purpose of this particular set of OGSEs is to provide a very precise reference illumination source for alignment and testing of the high-resolution spectrographs that are part of ESA's SENTINEL-5/UVNS Instrument onboard the MetOp SG Satellite, in the context of the Copernicus Earth Observation Programme.

The Sentinel-5 Mission [1] is dedicated to monitoring trace gas concentrations and aerosols in the atmosphere in a daily, near-real time basis. Data generated by the mission will be crucial and extremely useful for scientists and governments worldwide to assess climate and air-quality protocols.

The Sentinel-5 instrument consists in five spectrometers covering the spectral range from the UV to the SWIR. The instrument comprises two wide FoV telescopes which collect the necessary light for the spectrometers. The slits in the Sentinel-5 instrument have a new design in which they act as slit homogenizers. This allows to scramble the scene in spectral direction which makes the spectral response of instrument less sensitive to heterogeneous scenes. The slit homogenizer is a planar light guide, formed by two parallel mirror plates.

To maintain the spatial content in the spatial direction, the spectrometers have an astigmatic collimating optics. This astigmatic optics have two object planes, located at the entrance and at the exit of the slit homogenizer. As such, to perform the optical characterization of the spectrometers, the OGSE must provide an astigmatic beam, where the distance between

*rhenriques@lusospace.com 
the two focal planes is equal to the homogenizer length, and the focal planes are located at the spatial and spectral object planes of the spectrographs.

The optical performance requirements for the OGSEs are obviously very strict. This makes the tasks of alignment, characterization, and optical testing yet more crucial, with stringent verification budgets. And such tasks rely, not only, but heavily, on the procedures described here. The paper will focus on the alignment task in particular.

\section{BRIEF DESCRIPTION OF SENTINEL-5 AIT OGSES}

LusoSpace is responsible for the design, manufacturing, assembly and testing of a subset of the S5 AIT OGSEs: ILL OGSE and ILL GSS OGSE. They are currently being used for alignment and verification tests of the S5 spectrometers in ISO 5 conditions and thermal vacuum environment. Three sets of OGSEs were developed, for the UV1, UV2VIS and NIR spectral ranges, with similar design concepts. Figure 1 shows a top view photograph of part of the S5 UV1 AIT OGSE, where two OGSEs (ILL UV1 and ILL GSS UV1) modules are assembled in a common baseplate. Several other components along the fiber paths that lead to the assembly shown in Figure 1 are also considered part of the OGSE but are not depicted here.

The ILL OGSE provides 7 point sources with divergence defined by rectangular masks. The ILL GSS OGSE images the light of a pinhole onto two different image planes, providing an astigmatic beam with divergence also defined by a rectangular mask. The masks, in both cases, define a non-equal divergence in the spatial $(X)$ and spectral $(Y)$ directions.

While the ILL OGSE design is based on a fixed block and does not require any alignment, the ILL GSS OGSE was conceived with the necessary opto-mechanical degrees of freedom (DOF) to enable alignment. This allows to ensure the set of the required characteristics of the output astigmatic beam - spatial and spectral F-numbers, and distance between the two foci. In addition, as an interface requirement between the two OGSEs, the ILL GSS OGSE beam propagation vector needs to be aligned with the beam vector of the ILL OGSE central field (F4 in Figure 1), which enforces two additional optimization parameters - ILL GSS pointing wrt ILL central (F4) pointing in spatial direction, and ILL GSS pointing wrt ILL central (F4) pointing in spectral direction.

The OGSE components are indicated in Figure 1. The main components of the ILL GSS module are a pinhole illuminated by a fiber (FP), a curved mirror (CM) and a rectangular aperture mask (AM). A folding mirror (FM) was included to make the design more compact. The astigmatism of the generated beam is mainly influenced by the relation between its angle of incidence at the $\mathrm{CM}$ and the radius of curvature of the $\mathrm{CM}$. For its alignment, the pinhole-fiber set has three linear DOF and the curved mirror has one angular DOF. This will be further detailed and summarized in the next section.

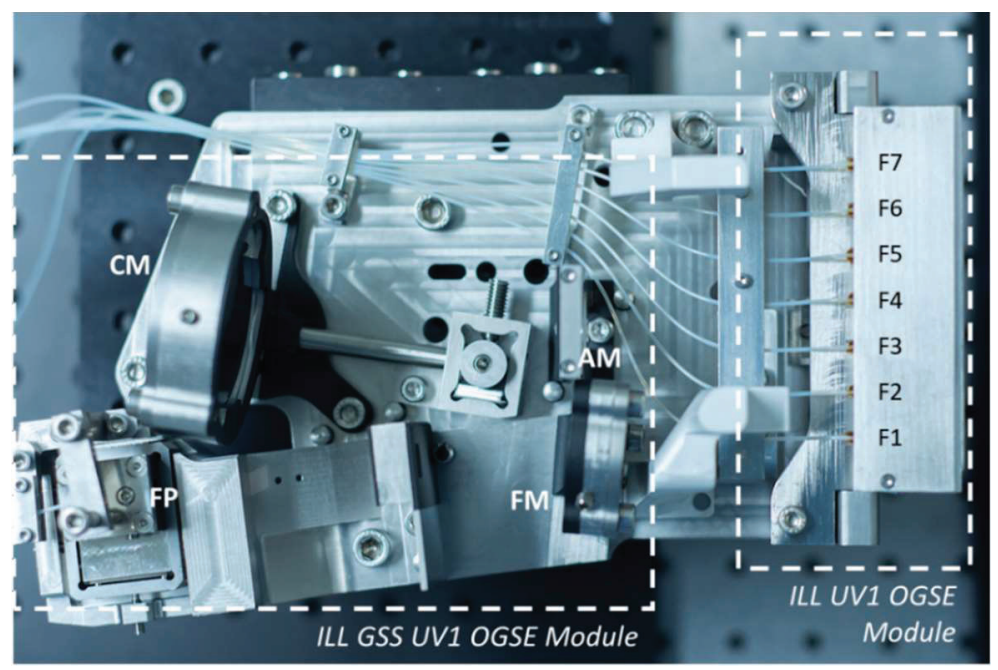

Figure 1 - Sentinel-5/UVNS UV1 AIT OGSE. Two OGSEs (ILL UV1 and ILL GSS UV1) modules are assembled in a common baseplate. 


\section{GENERAL DESCRIPTION OF OGSE ALIGNMENT}

This work focus the attention on the OGSE alignment procedure task. For a clear understanding of such task, Figure 2 shows a flow chart with the procedural steps, while Table 1 lists the parameters to be optimized (Optimization Parameters) and the opto-mechanical DOFs to be adjusted with shims for that goal (Alignment Parameters).

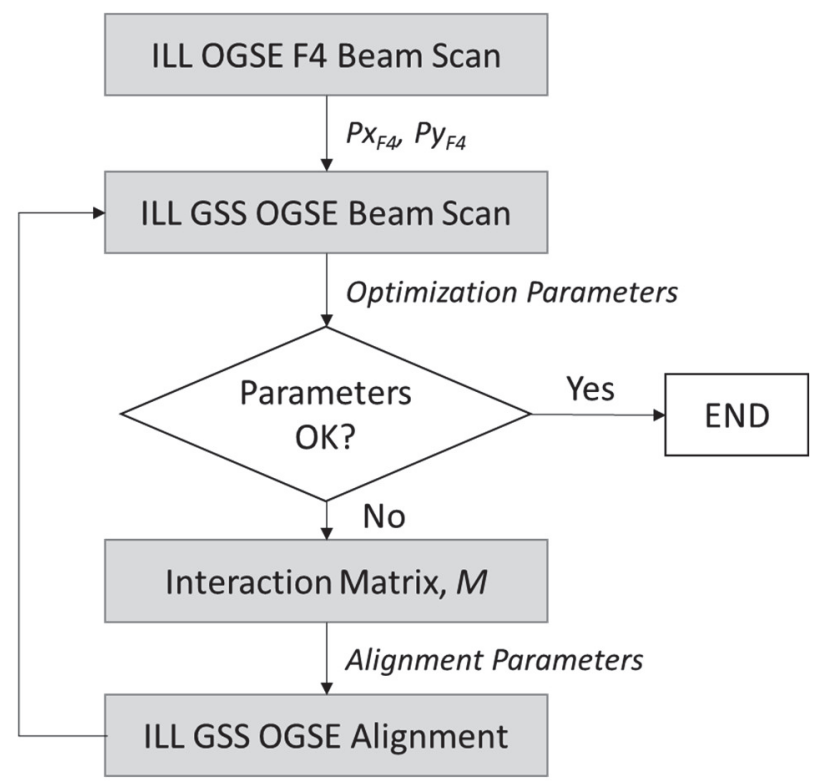

Figure 2 - ILL GSS OGSE alignment steps.

Table 1. OGSE alignment procedure: list of output parameters to be optimized (Optimization Parameters) and degrees of freedom available to achieve optimization (Alignment Parameters, see Figure 3).

\begin{tabular}{|c|c|}
\hline \multicolumn{2}{|r|}{ Optimization Parameters } \\
\hline Symbol & Description \\
\hline$F \#_{X}$ & ILL GSS F-number in spatial direction \\
\hline$F \#_{Y}$ & ILL GSS F-number in spectral direction \\
\hline$L_{\text {Ast }}$ & $\begin{array}{l}\text { Distance between spatial and spectral foci of ILL GSS - defined as the } \\
\text { astigmatism of the system }\end{array}$ \\
\hline$P_{X}^{\prime}=P_{X}-P_{X_{F 4}}$ & ILL GSS pointing wrt ILL central pointing in spatial direction (relative pointing) \\
\hline$P_{Y}^{\prime}=P_{Y}-P_{Y_{F 4}}$ & ILL GSS pointing wrt ILL central pointing in spectral direction (relative pointing) \\
\hline \multicolumn{2}{|r|}{ Alignment Parameters } \\
\hline Symbol & Description \\
\hline$x$ & $X$ position of fiber-pinhole set \\
\hline$y$ & $Y$ position of fiber-pinhole set \\
\hline$z$ & $Z$ position of fiber-pinhole set \\
\hline$t_{y}$ & Curved mirror tilt angle about $Y$ axis \\
\hline
\end{tabular}


Following the flow of Figure 2: the alignment procedure starts with the characterization of the ILL OGSE central beam (beam F4) to obtain its pointing information $\left(P_{X_{F 4}}\right.$ and $\left.P_{Y_{F 4}}\right)$, which will be used as pointing reference for the ILL GSS OGSE alignment. This beam characterization relies on an imaged-based method (referred to as Beam Scan in Figure 2), from which beam parameters are extracted by analyzing its evolution along a $Z$ scan. The experimental test setup and the imaging processing algorithm used to extract such beam performance parameters are explained in Section 4.

After obtaining the pointing reference, the ILL GSS optical beam is characterized with the same Beam Scan method, resulting in evaluation of $F \#_{X}, F \#_{Y}, L_{A s t} P_{X}, P_{Y}$. At this point, the optimization Parameters listed in Table 1 are fully known, at least for the current alignment iteration.

Optimization parameters values are then analyzed to check if they are close to the final target (requirement) values. If all the parameters are within the target budget within their evaluation uncertainty values, the alignment of the OGSE can be considered complete. If not, the parameters are used as input for the interaction matrix, $M$. This matrix describes the functional relation between the optimization parameters and the alignment parameters (DOFs of the system).

From the interaction matrix $M$, the corrections that must be imposed to each DOF in order to align the optical system, according to the alignment goals, are calculated. Section 5 describes in more detail how the matrix is used and how it was determined. After implementing the alignment suggestions from the matrix $M$ through shim adjustment in the closest dimensions available, the ILL GSS OGSE beam is scanned again, and the optimization parameters are reassessed. If all of them are within the defined budget, the alignment of the OGSE stops; if not, the parameters are again used as input for the interaction matrix, $M$ and a new solution for the alignment parameters is found. This methodology flow is repeated until all alignment requirements are achieved.

To better understand how the adjustment parameters are controlled, Figure 3 shows the ILL GSS module optical layout and its DOFs. Their adjustment is achieved through shims on dedicated mechanical bases of each component (FP or CM).

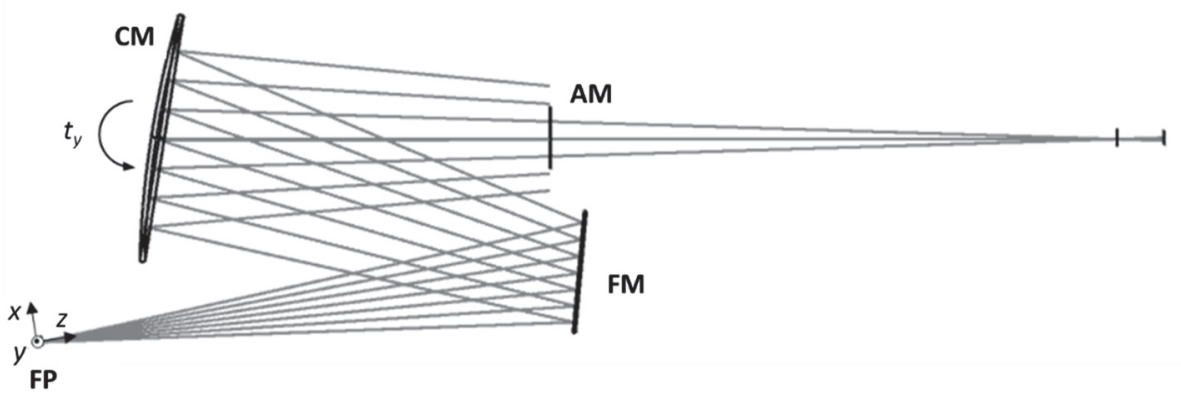

Figure 3 - ILL GSS OGSE module optical layout. There are 4 DOF: 3 linear at FP and one angular at CM.

\section{BEAM SCAN METHOD}

The beam scan method used as part of the overall alignment procedure (seeFigure 2) is described in this section. It is the core of the process, and keeping its uncertainties to very low values is essential for a precise OGSE alignment and accurate performance verification.

The method consists of acquiring a set of images along the optical beamline, by scanning the optical beam axially with a CCD sensor, and then evaluating from those images - via a dedicated image processing algorithm - the relevant optical optimization parameters. The method serves three purposes: to test the final optical performance of ILL OGSE and ILL GSS OGSE at test campaign phases, to assist in the characterization of the reference frames of both OGSEs, and to assist the alignment procedure of ILL GSS OGSE. This paper is focusing on the last one. 


\subsection{Experimental Procedure}

The CCD camera module is fixed on top of a computer-controlled precision 2-axis linear stage (Figure 4). This measurement set is, in turn, assembled in front of the OGSE exit pupil, around the $X$ location in which both reference beam F4 and the astigmatic beam can be imaged. After a dedicated pre-alignment procedure (not described here), a scan along the beam path will allow to characterize the evolution of the beams.

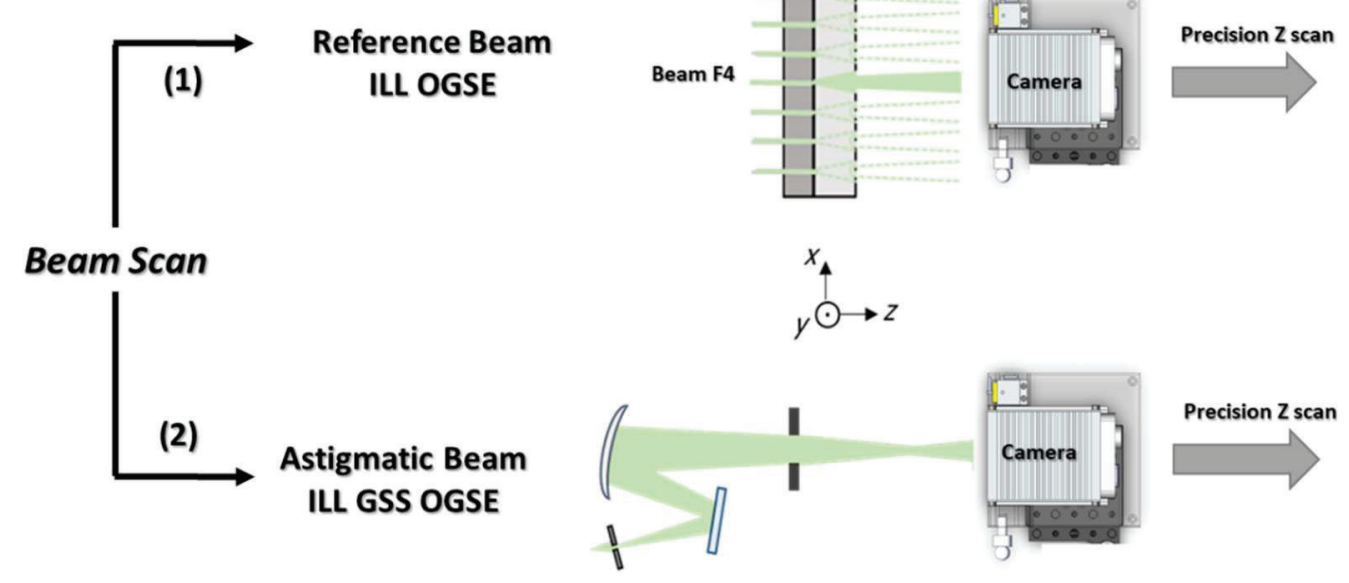

Figure 4 - Schematic representation of Beam Scan setup and procedure. The CCD camera module is mounted on a 2-axis precision motorized linear stage. The sensor is pre-aligned (its normal vector wrt beam F4) in a dedicated pre-procedure not described here. The front interface of the OGSE outputs the seven beams of ILL OGSE and also has a dedicated opening to allow proper output of ILL GSS OGSE astigmatic beam.

In the ILL GSS OGSE, the alignment verification implies that the ideal starting position for the beam scan is such that the beam is already diverging after both foci. Imaging the foci can be useful for other performance testing aspects, but the concentration of light in very few pixels severely undermines centroid detection accuracy via image processing.

The locations of both foci are determined using the beam size evolution along Z. The first focus of the ILL GSS is located where the beam has its minimum size in the spatial direction $(\mathrm{X})$, while the second focus corresponds to the minimum beam size in spectral direction $(\mathrm{Y})$. Both locations are calculated by the intersection of the beam's width/height linear fit with a constant close to zero.

Because the beams are diverging through the scan, sensor exposure time is automatically controlled within an internal loop to keep saturation level (and hence border contrast levels) constant along the scan.

\subsection{Image Processing Algorithm}

\section{High-Level Description}

A dedicated algorithm with different processing modules takes the scan image data set analyze it. In a high-level general description:

- Image Pre-processing;

- Derivative-based sub-pixel border detection algorithm;

- Note: both diffraction and remaining speckle effects are important here and must be carefully considered in algorithm definitions as to produce the most accurate and physical-sound results. Moreover, algorithms were developed in a way that output beam parameters are not affected by the exact location of the axial region defining that particular scan. 
- In-plane evaluation of beam dimensions and centroid $X-Y$ position;

- Loop through all images in the scan generates plots of beam width, height, and centroid position versus axial $Z$ position;

- Data linear fits;

- Evaluation of parameters of interest listed in Table 1 from the linear fits and position knowledge.

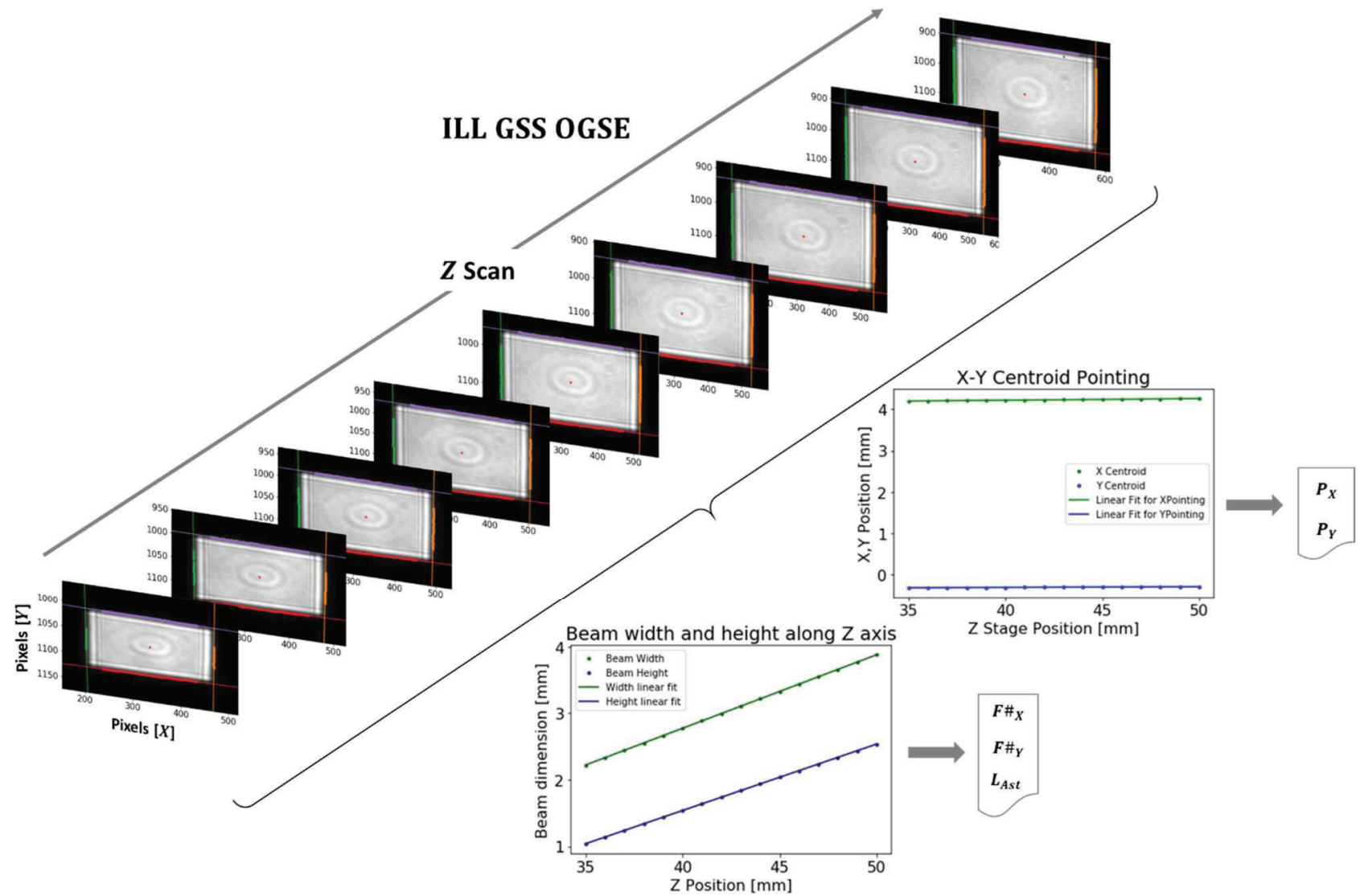

Figure 5. Schematic depiction of beam scan acquisition results and processing, for the astigmatic beam. An analogous process is carried out previously for the reference beam F4, leading to outputs $F \#_{X_{F 4}}, F \#_{Y_{F 4}}, P_{X_{F 4}}$, and $P_{X_{F 4}}$, the last two being then used for calculating the relative pointing between reference and astigmatic beams, $P^{\prime}{ }_{X}$ and $P_{Y}^{\prime}$ respectively, in $X$ and $Y$ directions. Note: For visualization purposes, not all images from a typical scan are depicted (usually a small $\Delta Z$ step of the order of $100 \mu \mathrm{m}$ is used).

\section{Uncertainty Analysis}

Measurements described here combine in-plane $(X-Y)$ image processing uncertainties of different parameters, in pixels or fraction of pixels, with linear stage displacement uncertainty, and the resulting uncertainty in the corresponding linear fits. The estimated uncertainty in $F \#$ evaluation, for example, must take into account beam dimensions (width $W$ and height $H$ ) uncertainties, plus the uncertainty in the knowledge of the axial $(Z)$ position. As for the uncertainty in beam lateral dimensions $W$ and $H$, with sub-pixel accuracy border detection algorithms such as the ones used here, and with good control of image acquisition conditions (total dark background, uniform beam image saturation along the whole scan, etc), we can conservatively estimate it to be $\delta W=\delta H \approx 2 \times 0.5$ pixel. 
By combining the linear stage accuracy and propagating the uncertainties, a final theoretical estimation for the relative error in $F \#$ is then given by

$$
\frac{\delta F \#}{F \#}=\sqrt{\left(\frac{\delta W}{W}\right)^{2}+\left(\frac{\delta \Delta Z}{\Delta Z}\right)^{2}} .
$$

For beam pointing $(P)$ verification, a similar estimate applies,

$$
\frac{\delta P}{P}=\sqrt{\left(\frac{\delta C}{C}\right)^{2}+\left(\frac{\delta \Delta Z}{\Delta Z}\right)^{2}}
$$

where $C=\left(C_{X}, C_{Y}\right)$ is the in-plane centroid position for each $\mathrm{Z}$ stage position, with $C_{X}$ along the horizontal beam dimension and $C_{Y}$ along the vertical. $\delta C / C$ is a quantity analogous to $\delta W / W$, both being directly proportional to the difference between two sub-pixel evaluated $X$ or $Y$ locations in each border of the beam.

Then, on these set of data points, linear fits along $Z$ account for further uncertainty reduction - proportional to the square root of the number of images $N$ in the dataset - while also averaging out possible $Z$ wobble effects of the linear stage.

Lastly, with the position of each focal point of the astigmatic beam being a geometrical extrapolation of the line equations of beam width $W$ and beam height $H$ versus $Z$, the uncertainty in $\delta L_{A s t}$ comprises the estimated error in both angular and linear coefficients of the fitted lines.

Table 2 presents the theoretical values estimated for the uncertainties in the output beam parameters evaluated from the beam scan imaging method, along with the corresponding uncertainty values obtained at a later stage from experimental repeatability analyses.

Table 2 - Uncertainty analysis for beam optimization parameters.

\begin{tabular}{|c|c|c|}
\hline \hline \multicolumn{3}{|c|}{ Estimated Uncertainties } \\
\hline \hline Parameter & Theoretical & From repeatability \\
\hline $\boldsymbol{\delta} \boldsymbol{F} \#_{\boldsymbol{X}}$ and $\boldsymbol{\delta} \boldsymbol{F} \#_{\boldsymbol{Y}}$ & \pm 0.01 & \pm 0.02 \\
\hline $\boldsymbol{\delta} \boldsymbol{L}_{\boldsymbol{A s t}}$ & $\pm 6 \mu \mathrm{m}$ & $\pm 10 \mu \mathrm{m}$ \\
\hline $\boldsymbol{\delta} \boldsymbol{P}^{\prime}{ }_{\boldsymbol{X}}$ and $\boldsymbol{\delta} \boldsymbol{P}_{\boldsymbol{X}}^{\prime}$ & $\pm 6 \operatorname{arcsec}$ & $\pm 12 \operatorname{arcsec}$ \\
\hline
\end{tabular}

\section{INTERACTION MATRIX $M$ AND SOLUTION OF THE SYSTEM OF EQUATIONS}

\subsection{Mathematical Model}

During the alignment of the optical system, deviations of the optimization parameters from their design values can be related with displacements between the actual position and the design position of the elements of the optical system [2]. Assuming that the optimization parameters are linearly (or casi linearly) dependent with the misalignment of the optical components, the relation between the set of optimization parameters $(F)$ and the alignment parameters $(D)-$ or more specifically their departure from nominal values, $\Delta F$ and $\Delta D$, respectively - can be expressed as

$$
\Delta F=A \Delta D
$$

where

$$
\Delta F=\left[\begin{array}{c}
\Delta F \#_{X} \\
\Delta F \#_{Y} \\
\Delta L_{A s t} \\
\Delta P_{X}^{\prime} \\
\Delta P_{Y}^{\prime}
\end{array}\right]=\left[\begin{array}{c}
F \#_{X}{ }^{m} \\
F_{Y}{ }^{m} \\
L_{A s t}{ }^{\prime} \\
P_{X}^{\prime}{ }_{X} \\
P_{Y}^{\prime}{ }^{m}
\end{array}\right]-\left[\begin{array}{c}
F \#_{X}{ }^{d} \\
F \#_{Y}{ }^{d} \\
L_{A s t}{ }^{d} \\
P_{X}^{\prime}{ }^{d} \\
P_{Y}^{\prime}{ }^{d}
\end{array}\right], \quad A=\left[\begin{array}{ccc}
\frac{\partial F \#_{X}}{\partial x} & \cdots & \frac{\partial F \#_{X}}{\partial t y} \\
\vdots & \ddots & \vdots \\
\frac{\partial P_{X}^{\prime}}{\partial x} & \cdots & \frac{\partial P_{X}^{\prime}}{\partial t_{y}}
\end{array}\right], \quad \Delta D=\left[\begin{array}{c}
\Delta x \\
\Delta y \\
\Delta z \\
\Delta t_{y}
\end{array}\right]=\left[\begin{array}{c}
x^{m} \\
y^{m} \\
z^{m} \\
t_{y}{ }^{m}
\end{array}\right]-\left[\begin{array}{c}
x^{d} \\
y^{d} \\
z^{d} \\
t_{y}{ }^{d}
\end{array}\right]
$$


$\Delta F$ is the difference between the measured $\left(F \#_{X}{ }^{m}\right.$, for example) and desired value $\left(F \#_{X}{ }^{d}\right.$, for example) of the optimization parameters. $A$ is the sensitivity matrix, which was obtained by simulation of the optical system, and $\Delta D$ represents the misalignments responsible for the deviation of the optimization parameters and that need to be corrected.

The alignment parameters can be determined by solving equation (3). The system is an overdetermined one since it has more optimization parameters than variables. This system can be determined as

$$
\Delta D=\left(A^{T} A\right)^{-1} A^{T} \Delta F .
$$

The misalignments of the system are determined by

$$
\Delta D=M \Delta F,
$$

where $M$ is interaction matrix defined as

$$
M=\left(A^{T} A\right)^{-1} A^{T}
$$

\subsection{Evaluation of Sensitivity Coefficients}

The calculation of the interaction matrix Mimplies determine the sensitivity of each optimization parameter with the alignment parameters, which was obtained by ray tracing simulation of the optical system. Figure 6 shows the relations between the optimization parameters and the displacement of the optical elements of the system, which are all approximately linear. However, such linear approximations are valid for small displacements only. This means that the components of the optical system need to be relatively close to their design positions at the start of the alignment process, for this method to be reliable. 

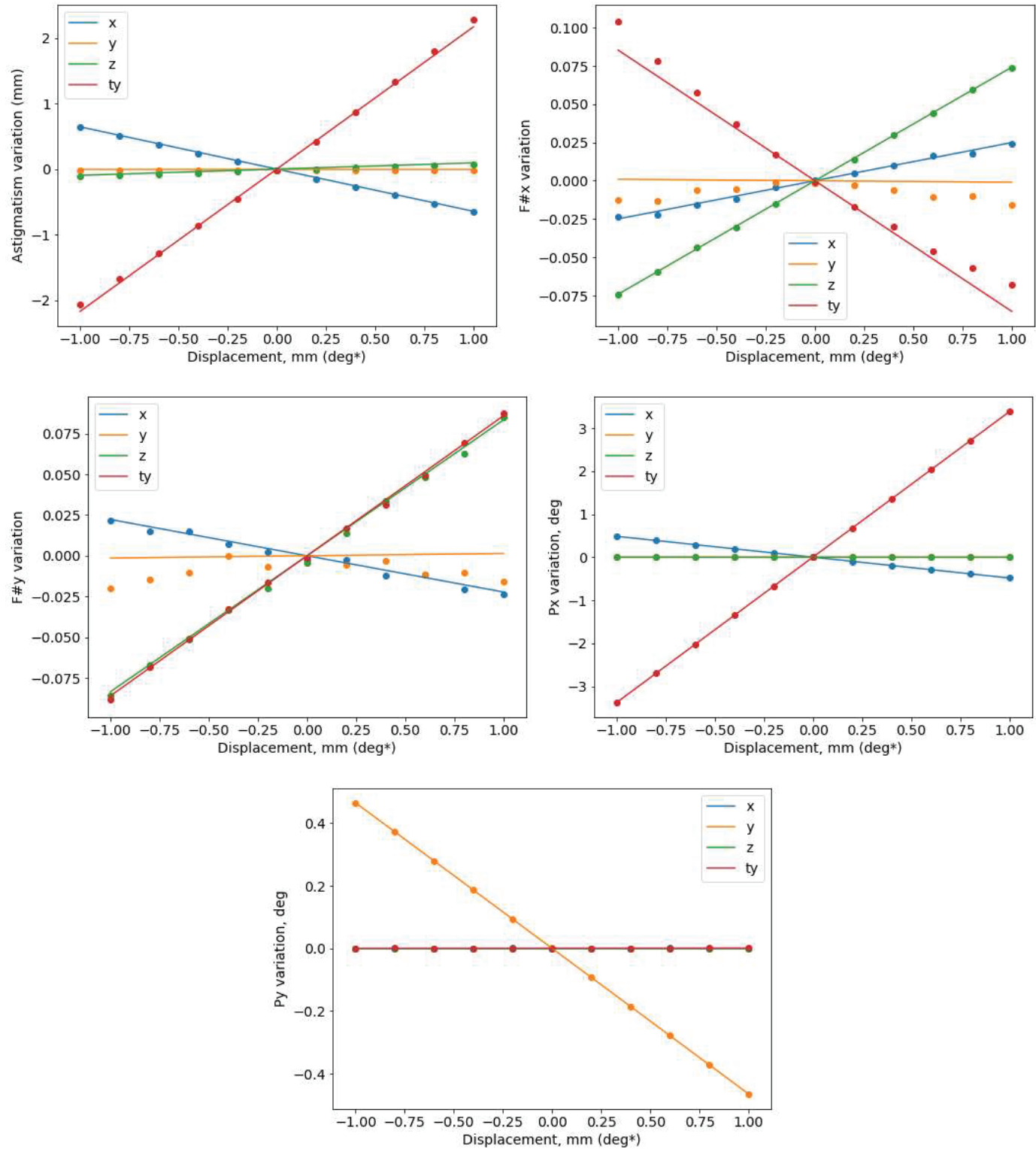

Figure 6. Relation between the optimization parameters with the displacement of optical elements. *Displacement units in $\mathrm{mm}(x, y$ and $z)$, and in $\operatorname{deg}\left(t_{y}\right)$.

\section{TEST CASE RESULTS}

\subsection{Simulation Alignment Results}

The described alignment method was tested by ray tracing simulation of the optical system. Twenty different configurations were tested: each configuration has a different starting misalignment. Figure 7 shows the simulated alignment performance results with only one interaction. As the results show, after one alignment interaction, all the performance parameters have reached their target within the measurement accuracy (Table 2). The spatial and spectral F\# are the parameters with higher dispersions after alignment (Table 3); they are, nevertheless, within their measurement accuracy of 0.02 (Table 2). 

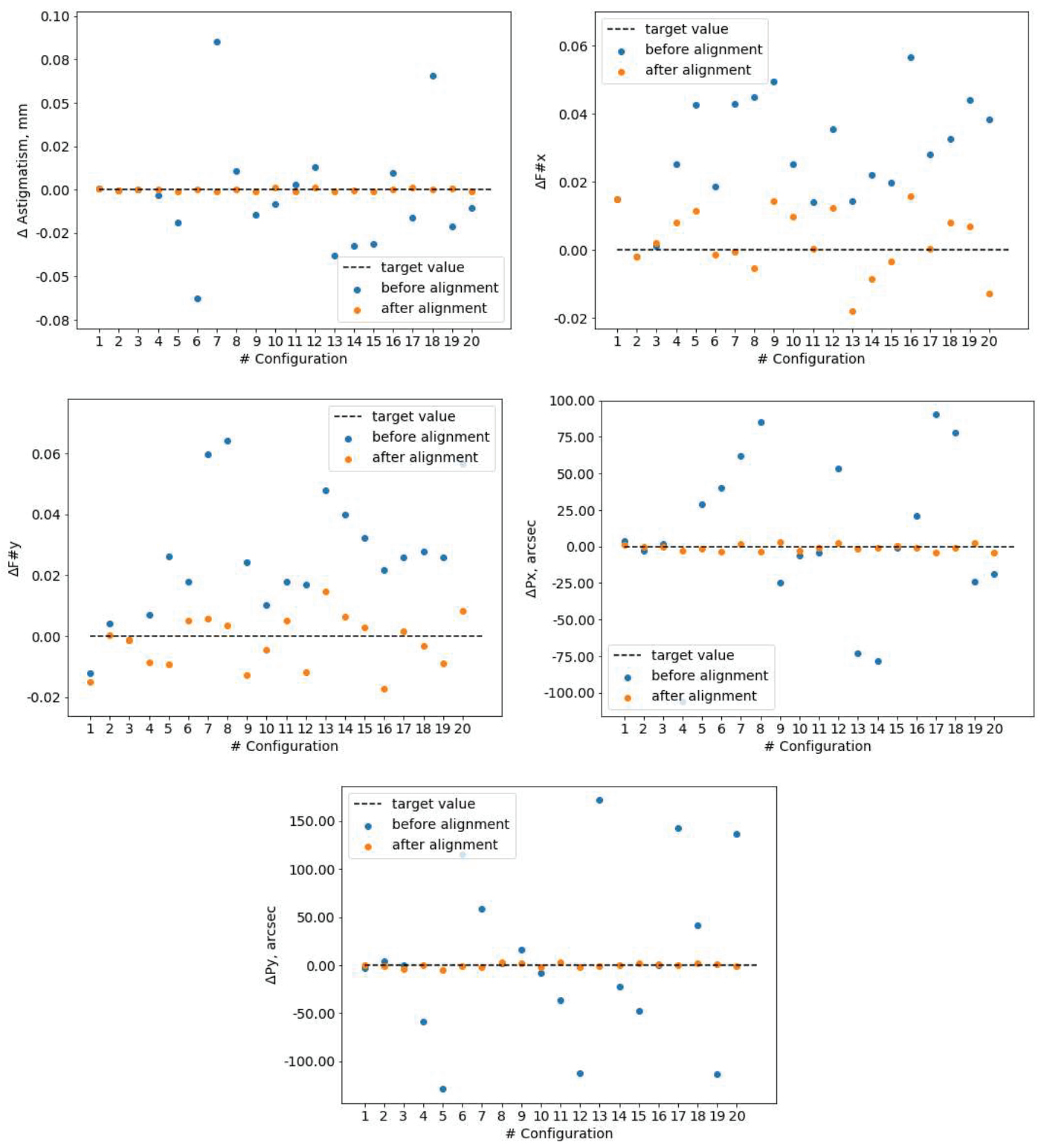

Figure 7. Simulated alignment performance (one iteration) results for 20 sets with different starting alignment configurations.

Table 3. Optimization parameters average and standard deviation before and after the alignment for 20 simulated test cases.

\begin{tabular}{|c|c|c|c|c|}
\hline \hline \multirow{2}{*}{ Parameter } & \multicolumn{2}{|c|}{ Before Alignment } & \multicolumn{2}{c|}{ After Alignment } \\
\cline { 2 - 5 } & Average & Std & Average & Std \\
\hline \hline$\Delta \boldsymbol{F} \#_{\boldsymbol{X}}$ & 0.028 & 0.016 & 0.003 & 0.001 \\
\hline$\Delta \boldsymbol{F} \#_{\boldsymbol{Y}}$ & 0.026 & 0.020 & -0.002 & 0.009 \\
\hline$\Delta \boldsymbol{L}_{\text {Ast }}(\mathbf{m m})$ & -0.003 & 0.032 & 0.000 & 0.009 \\
\hline$\Delta \boldsymbol{P}_{\boldsymbol{X}}{ }_{\boldsymbol{X}}$ (arcsec) & 6 & 52 & -1 & 2 \\
\hline$\Delta \boldsymbol{P}_{\boldsymbol{Y}}{ }_{\boldsymbol{Y}}$ (arcsec) & 8 & 82 & 0 & 2 \\
\hline
\end{tabular}




\subsection{Experimental Alignment Results}

The alignment method was used to align all ILL GSS OGSE modules in the context of the project. Figure 8 shows the FAT campaign of the UV1 OGSEs at LusoSpace's ISO 5 cleanroom. The results of the experimental alignment process of the ILL GSS OGSE is shown in Table 4. The optimization parameters of the misaligned system were determined using the beam scan method described in Section 4 and used to determine the alignment parameters as shown in section 5 . After aligning the system by using the closest available set of shims as calculated by the interaction matrix $M$, the beam of the OGSE was scanned again and the new optimization parameters were determined. Since these parameters were inside the target budget, the optical system was considered aligned.

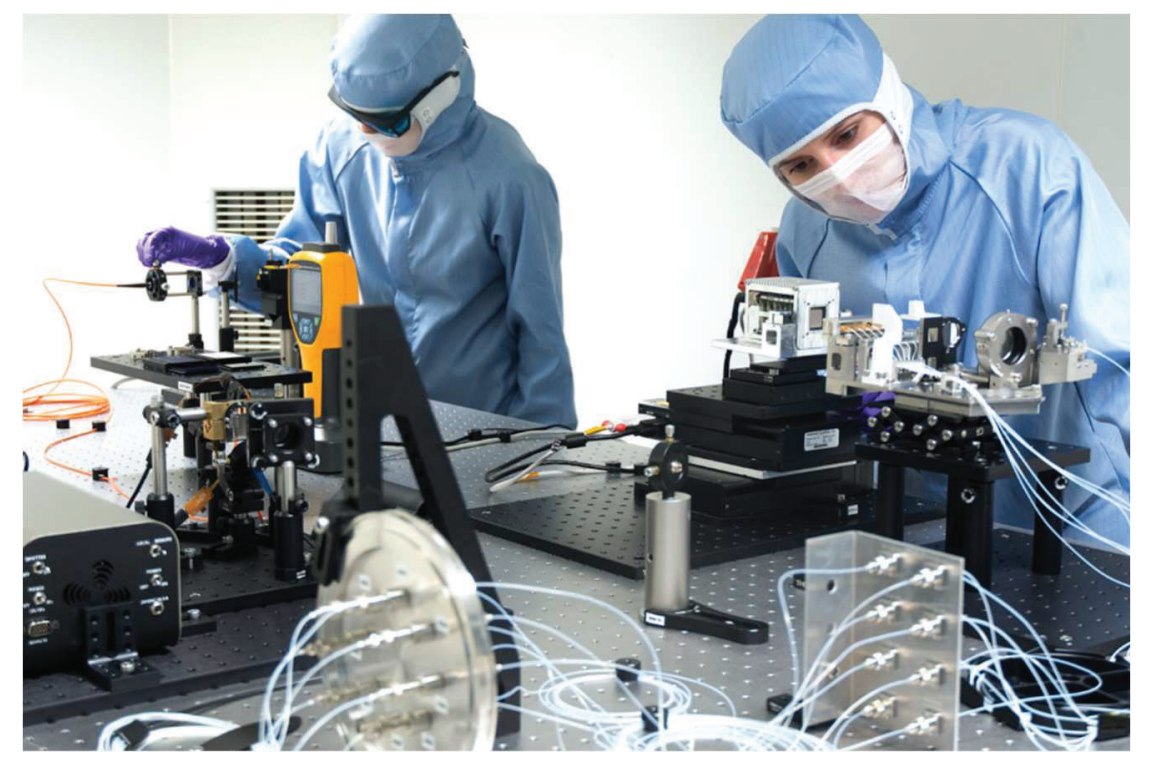

Figure 8. Test campaign of UV1 OGSEs at ISO 5 cleanroom in LusoSpace's facilities. The methods described in the paper were used to align and characterize the optical properties of the OGSEs.

Table 4. ILL GSS OGSE experimental alignment results showing optimization parameters before alignment, calculated and input alignment parameters, and optimization parameters after alignment.

\begin{tabular}{|c|c|c|c|c|}
\hline \multicolumn{5}{|c|}{ Optimization Parameters - Before Alignment } \\
\hline$\Delta \boldsymbol{F} \#_{X}$ & $\Delta \boldsymbol{F} \#_{Y}$ & $\Delta L_{\text {Ast }}(\mathrm{mm})$ & $\Delta P_{X}^{\prime}(\operatorname{arcsec})$ & $\Delta P_{Y}^{\prime}(\operatorname{arcsec})$ \\
\hline 0.12 & 0.05 & 0.01 & 1093 & -30 \\
\hline \multicolumn{5}{|c|}{ Alignment Parameters } \\
\hline & $x(\mathbf{m m})$ & $y(\mathbf{m m})$ & $z(\mathbf{m m})$ & $t_{y}(\operatorname{arcsec})$ \\
\hline Calculated & -0.304 & -0.016 & 1.063 & 480 \\
\hline Input & -0.30 & 0.00 & 1.00 & 516 \\
\hline \multicolumn{5}{|c|}{ Optimization Parameters - After Alignment } \\
\hline$\Delta F \#_{X}$ & $\Delta F \#_{Y}$ & $\Delta L_{\text {Ast }}(\mathbf{m m})$ & $\Delta P_{X}^{\prime}(\operatorname{arcsec})$ & $\Delta P_{Y}^{\prime}(\operatorname{arcsec})$ \\
\hline 0.03 & 0.01 & 0.00 & -21 & -67 \\
\hline
\end{tabular}




\section{SUMMARY AND CONCLUSIONS}

In this paper, an image-based beam analysis method used for optical alignment, characterization and testing of the Sentinel-5 AIT OGSEs was described. A general view on the experimental setup and procedure, and on the image processing algorithm used for beam characterization was presented. The method was shown to lead to measurement accuracies - based on actual experimental repeatability - of \pm 0.02 for spatial and spectral F-numbers, $\pm 10 \mu \mathrm{m}$ for the distance between foci of the astigmatic beam, and \pm 12 arcsec for relative beam pointing.

This beam scan method was then used in the alignment phase of the ILL GSS OGSE module, to determine the alignment parameters - or opto-mechanical degrees of freedom which would allow to obtain the final desired alignment and beam performances.

The complete alignment method is based on the beam scan method combined with a matrix representation of the sensitivity coefficients of the optical system obtained by simulation.

Both simulated and final experimental OGSE alignment results have proven the efficiency and practicality of the presented method.

The AIT OGSEs was recently successfully tested and delivered.

\section{REFERENCES}

[1] J. Irizar, M. Melfa, P. Bartsch, J. Koehle, S. Weiss, R. Greinacher, M. Erdmann, V. Kirschner, A. Perez Albinana, D. Martin, "Sentinel-5/UVNS", Proceedings of SPIE: International Conference on Space Optics. Vol. 11180 (2018)

[2] Wu, W., Shang, J., Cao, Y., Chen, C. and Zhou, J., "Research on Applicability of Sensitivity Table Method in Optical System Alignment”, IOP Conference Series: Earth and Environmental Science. 440, 032027 (2020). 